\title{
Characteristics and Driving Mechanism of Soil Organic Carbon Content in Farmland of Beijing Plain: Implication for the Fate of Engineered Polymers in Soil
}

\author{
Lanlan Zhang, ${ }^{1}$ Zhen Li $\mathbb{D}^{1},{ }^{1}$ Shiwen Zhang $\mathbb{D}^{2},{ }^{2}$ Shasha Xia, \\ Hongguang Zou, ${ }^{2}$ and Yuanfang Huang $\mathbb{B D}^{1}$ \\ ${ }^{1}$ College of Resources and Environmental Science, China Agricultural University, Beijing 100193, China \\ ${ }^{2}$ College of Earth and Environmental Sciences, Anhui University of Science and Technology, Huainan, China \\ Correspondence should be addressed to Shiwen Zhang; mamin1190@126.com and Yuanfang Huang; yfhuang@cau.edu.cn
}

Received 4 March 2019; Accepted 12 June 2019; Published 4 August 2019

Guest Editor: Tao Cheng

Copyright (c) 2019 Lanlan Zhang et al. This is an open access article distributed under the Creative Commons Attribution License, which permits unrestricted use, distribution, and reproduction in any medium, provided the original work is properly cited.

Soil organic matter (SOM), as a kind of natural polymers, affects the migration and transport of ions and particles in soil system due to its surface characteristics and interaction and then causes significant changes in soil quality such as soil fertility loss and pollutant transfer. It is of great importance to study the temporal and spatial evolution of soil organic matter and its driving mechanism for soil health management. This study aims to fully reveal the evolution characteristics and driving mechanism of soil organic carbon (SOC) in farmland of the Beijing plain based on a six-year site monitoring. According to the research results, there is a significant difference in the overall soil organic content during the 6-year period. The temporal stability of SOC is moderate, and it is inversely proportional to SOC content in terms of spatial distribution. SOC content increases as organic fertilizer input rises, and an extra unit $\left(15 \mathrm{~kg} \cdot \mathrm{ha}^{-1}\right)$ of organic fertilizer input leads to an increase of $0.057 \mathrm{~g} \cdot \mathrm{kg}^{-1}$ in SOC content. The soil with higher clay content exhibits higher SOC content. The organic carbon content in different soil texture types increases with time, and there is a significant difference between the increases in medium loam and light loam. The grain field plantation system exhibited declining SOC content, while the protected vegetable fields, open vegetable fields, and orchards all showed an increase in SOC content. According to our results, the SOC content of farmland in the plain areas of Beijing is largely dependent on the input of organic carbon if other conditions remain unchanged or exhibit insignificant changes.

\section{Introduction}

With the development of modern macromolecule chemical industry and the intensification of agricultural production, many synthetic organic compounds have been discharged into soil directly or indirectly through the atmosphere or water body in modern human production and life. Microplastics are widely used in remediation of contaminated soil due to their small size and large specific surface area $[1,2]$. However, due to the specific environmental behavior and potential environmental risks of engineering microplastics, many researchers believe that the use of engineering nanomaterials should be prohibited in in situ repair [3]. Therefore, more and more researchers focus on the nature and application of natural soil microplastics. Soil organic matter
(SOM), as an important source for natural microplastic in soil, participates in various ecological processes including element cycling and pollutant migration [4], because it has had large specific surface area and is easy to adsorb dissolved organic matter in soil-water. SOM can improve soil adsorption, buffering, and fertilizer retention and makes soil more loose, noncohesive, and easy to cultivate. In addition, it also can store carbon from the atmosphere and other pollutants and alleviates the biological hazards of chemical pesticides, heavy metals, and other pollutants in soil [5-7]. For example, if humic acid particles, a kind of important ingredient of soil organic matter, are adsorbed on the surface of microplastics, the steric hindrance of microplastics will increase, thus affecting the migration of microplastics $[8$, 9]. 
Therefore, it is of great significance and urgent to study the spatiotemporal changes and driving mechanisms of soil organic matter and to reasonably and effectively control the content of soil organic matter for maintaining soil fertility, reducing soil pollution, and promoting the sustainable utilization of soil resources.

In the natural environment, adsorption will affect the migration, transformation, and biodegradation of pollutants [10]. Surfactants are used to treat refractory organic pollutants in soil-water environment to promote special desorption of soil and increase apparent water phase concentration. Several studies have been carried out on the application of surfactants to strengthen remediation of specially polluted soil, including soil cleaning application and bioremediation $[11,12]$. SOC content has obvious characteristics of temporal evolution, and many studies have been conducted on characterizing the evolution of farmland SOC content [13-16]. Carbon simulation models have been adopted in some analyses of evolution features of regional SOC content. At present, the carbon models that are frequently used worldwide include DNDC [17], RothC [18], CENTURY [19], and APSIM [20]. All these models have been applied to simulate changes in farmland SOC content at both national and regional levels [13-16, 21]. The method of mathematical modeling has more advantages in simulating SOC dynamics of farmland in larger spatial and temporal scales. But the simulation results are not representative of the actual situation. Therefore, the study on SOC content change based on data from long-term monitoring at fixed locations is needed.

Research on the driving mechanism of SOC change based on sample points has been carried out. However, most of the factors used to analyze the driving mechanism are static, e.g., elevation and soil type which mainly affects the spatial distribution pattern of SOC content. The dynamic development of farmland SOC is also driven by the natural environment and artificial management. Human activities impose significant effects on SOC. According to Davidson and Ackerman [22], the organic carbon in the $30 \mathrm{~cm}$ surface layer of soil could be reduced by as much as $40 \%$ on average after natural vegetation is reclaimed into farmland. According to some studies [23, 24], improvements in agricultural management, such as minimum and no tillage, and the increase of chemical and organic fertilizers, can alleviate or reverse the loss of SOC. As a result, it is necessary to explore the impact of human activities on the change of soil organic carbon content.

In short, this study, taking the farmland of Beijing plain as the study area, aims to (1) reveal the evolution characteristics of SOC during 2010 to 2015 and (2) explore the influence of human activity like organic manure and planting system and soil properties such as soil texture on the change of SOC based on the long-term location monitoring data.

\section{Data and Methods}

2.1. Overview of the Research Zones. According to the digital elevation of Beijing and the spatial distribution of farmland, the $100 \mathrm{~m}$ contour was set as the research boundary. The research area is located in the eastern plain of Beijing, with a total area of about $7779.12 \mathrm{~km}^{2}$ (Figure 1). The research zones were divided into three functional areas, including cover the water source conservation area, the agricultural security area, and the urban life area. The area of light loam is the largest, followed by sandy loam and middle loam (Figure 2). And the international standard soil texture classification standard was adopted in this study.

2.2. Data Processing and Analysis. Long-term monitoring points have been established according to the distribution of farmland. There were 149, 137, 129, 123, 104, and 91 sampling points from 2010 to 2015 (Figure 1). To ensure the representativeness of the samples, the cross-section soil samples were all collected from the central points and the two neighboring angles at each sampling point. Furthermore, the soil samples collected at the same depth were mixed prior to testing (Figure 1). Potassium dichromate titration was adopted to assess SOC (Johnson, 2010). Aside from the data collected at the monitoring points, the digital elevation (resolution: $25 \mathrm{~m} \times 25 \mathrm{~m}$ ), the 1:50000 soil texture map, and the input of organic fertilizer were obtained via the Beijing municipal digital soil system and field investigation.

SPSS software (SPSS, Inc., Chicago, IL, USA, 2012) was applied to conduct descriptive statistical analysis and variance analysis. The temporal stability, the spatial prediction, and spatial correlation analysis were conducted in the ArcGIS 10.2 (ESRI, 2010).

\subsection{Study Methods}

2.3.1. Empirical Bayesian Kriging. The traditional linear kriging methods have strong smoothing effects and require a relatively large number of sampling points. Meanwhile, the data also needs to satisfy the spatial stationarity hypothesis. The empirical Bayesian kriging (EBK) method can measure unstable variables and is more accurate than other kriging methods for small data sets $[25,26]$. EBK estimates basic semivariation functions to describe the introduced errors. The input data is used to simulate several semivariation functions to describe the uncertainty of semivariation function estimation, and the predicted standard error should be smaller [25-28].

Additionally, we also validated whether the different number of samples in the above six years can meet the research requirements. Referring to the Technical Specification for Soil Environmental Monitoring, the variability coefficient and relative deviation are used to determine whether the sample points meet the research requirements. The formula is as follows.

$$
n=t^{2} \cdot \frac{C V}{m^{2}}
$$

where $n$ is the number of samples, $t$ is the value of $t$ at a certain degree of freedom at a given confidence level (generally $95 \%$ for soil monitoring), $C V$ is the coefficient of variation (\%) obtained from the collected data, $m$ is the acceptable relative deviation (\%), and soil monitoring is generally limited to $10-20 \%$. 

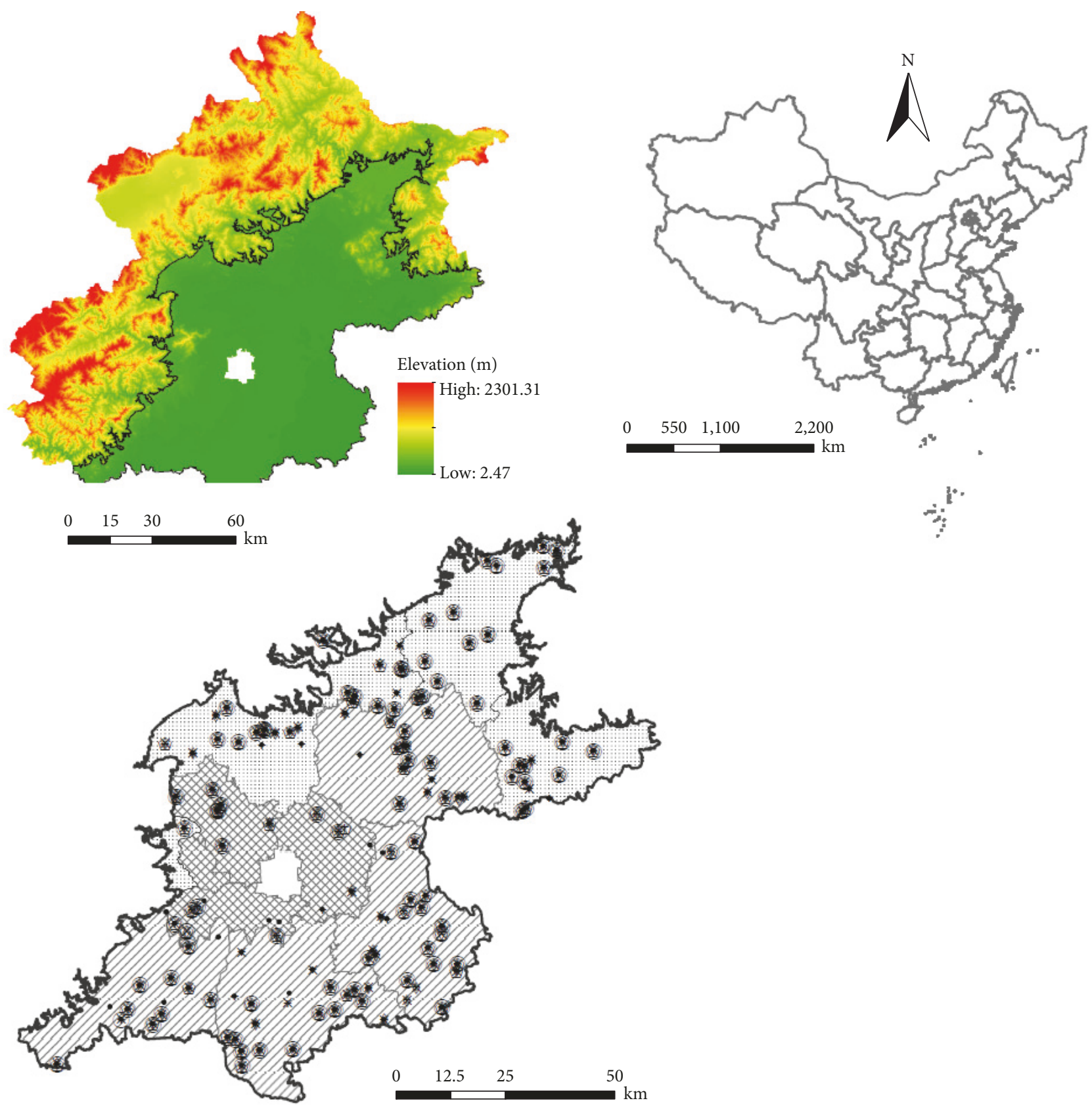

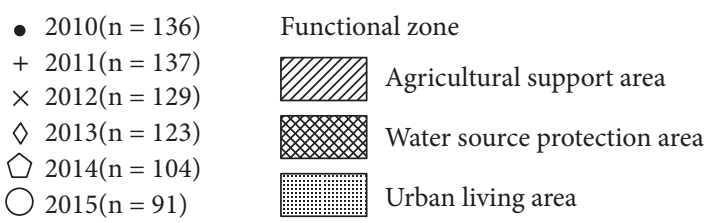

FIGURE 1: Map of the study area, sampling points, and functional areas.

In this study, the coefficient of variation $(\mathrm{CV})$ of SOC in the above six years was $39.31 \%, 33.87 \%, 47.02 \%, 40.62 \%$, and $48.57 \%$, respectively. According to the analysis results, the average coefficient of variation (\%) of all monitoring indicators was $42.03 \%$ and the acceptable relative deviation $\mathrm{m}$ was taken as the minimum value of $10 \%$. Through calculation, we need to set up sampling points 40 . Based on the above results, the minimum sampling points of 91 in 2015 can meet the research requirement. As a result, the number of samples in different years can meet the analysis requirements. 


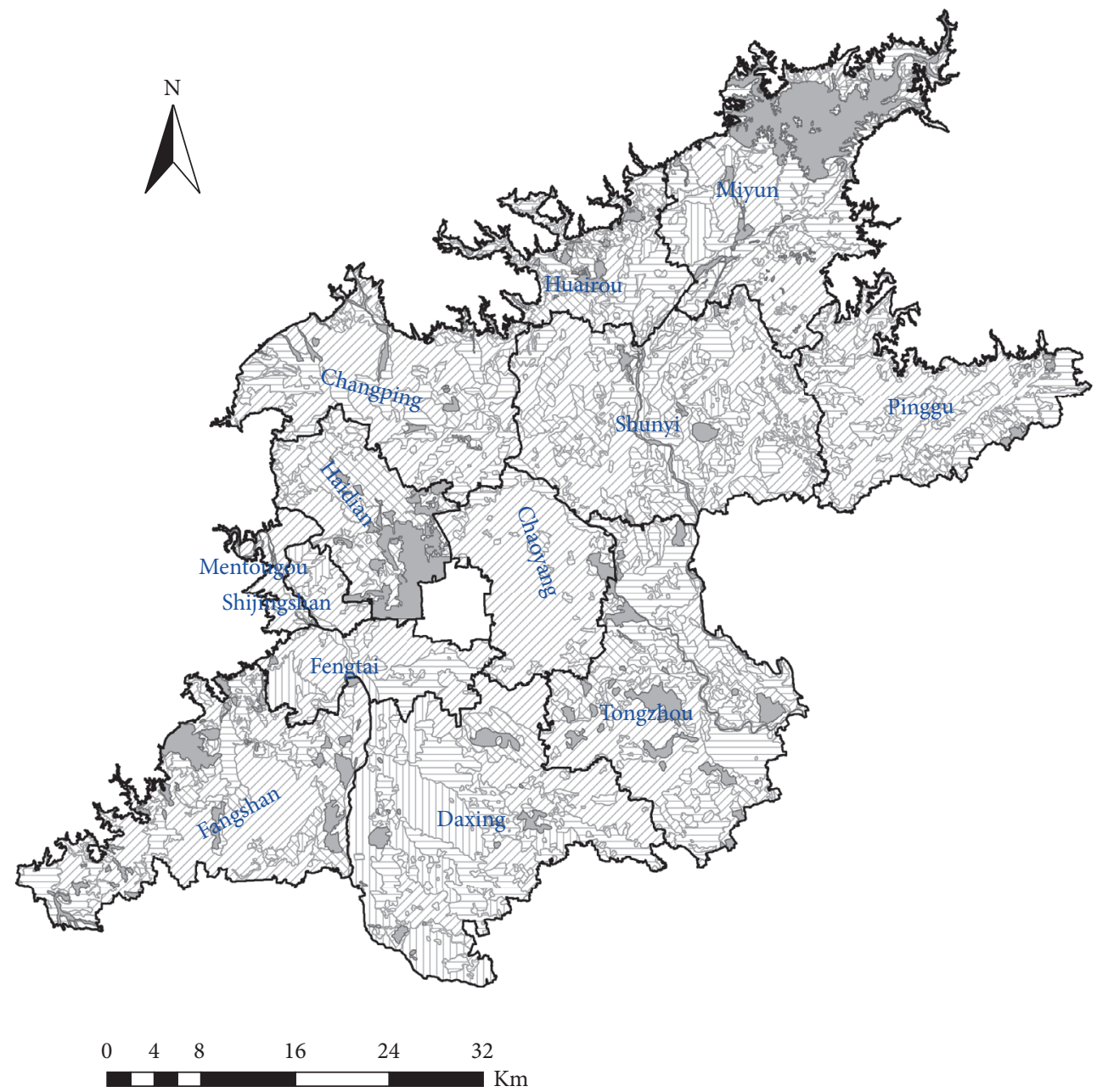

Soil texture

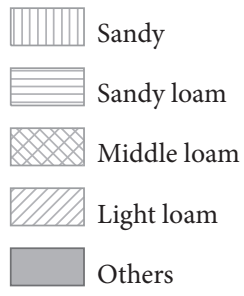

Figure 2: Map of soil texture in the study area.

2.3.2. Overall Temporal Stability Coefficient of SOC. The SOC temporal stability in the 6-year study period was quantified by the variation coefficients of measurements obtained from repeated sampling. This study adopts the variation coefficient calculation method first proposed by Blackmore [29]. The equation for the temporal stability coefficient $\left(T S C V_{t i}\right)$ is as follows:

TSCV $V_{\text {it }}$

$$
=\sqrt{\frac{\left(n \times \sum_{t=1}^{n} S O C_{i t}{ }^{2}-\left(\sum_{t=1}^{n} S O C_{i t}\right)^{2} / n \times(n-1)\right.}{\left(\sum_{t=1}^{n} S O C_{i t}\right) / n}}
$$

where $S O C_{i t}$ refers to the measured value of soil property obtained at the " $i^{t h}$ " sampling point at the " $t$ "th" sampling time, and " $n$ " is the frequency of sampling $(n=6)$.

The temporal stability coefficients were divided into five levels in three types, namely, "Stable" $(<10 ; S)$, "Medium Stability" (10-25; MS), and "Unstable" (>25; US). "Medium Stability" was further divided into three levels, namely, MS1 (10-15), MS2 (15-20), and MS3 (20-25) [29].

2.3.3. Quantitative Analysis of Relationships between SOC Content and Influencing Factors. Simple spatial correlation 
TABLE 1: Characteristic statistical values of SOC for different years.

\begin{tabular}{lcccr}
\hline Years & Number of samples & Mean $/ \mathrm{g} \cdot \mathrm{kg}^{-1}$ & Standard deviation $/ \mathrm{g} \cdot \mathrm{kg}^{-1}$ & $\mathrm{Rang} / \mathrm{g} \cdot \mathrm{kg}^{-1}$ \\
\hline 2010 & 136 & $10.699 \mathrm{~b}$ & 4.205 & 27.320 \\
2011 & 137 & $10.869 \mathrm{ab}$ & 3.681 & 21.920 \\
2012 & 129 & $10.475 \mathrm{~b}$ & 4.925 & 32.175 \\
2013 & 123 & $11.485 \mathrm{a}$ & 4.913 & 0.100 \\
2014 & 104 & $11.586 \mathrm{a}$ & 4.706 & 0.534 \\
2015 & 91 & $12.078 \mathrm{a}$ & 5.659 & 35.991 \\
\end{tabular}

analysis $(R)$ can be used to explore the relationship between two specific variables.

$$
R_{x y}=\frac{\sum_{i=1}^{n}\left[\left(x_{i}-\bar{x}\right)\left(y_{i}-\bar{y}\right)\right]}{\sqrt{\sum_{i=1}^{n}\left(x_{i}-\bar{x}\right)^{2} \sum_{i=1}^{n}\left(y_{i}-\bar{y}\right)^{2}}}
$$

where $R_{x y}$ is the spatial correlation coefficient of $x$ and $y, x_{i}$ is the SOC content in the " $i$ " " year, $y_{i}$ is the input of organic fertilizer in the " $i$ "th" year, $\bar{x}$ is the mean SOC content of six years, $\bar{y}$ is the mean organic fertilizer input of six years, and $n$ is the total number of years $(n=6)$.

\section{Results and Discussion}

3.1. Overall Evolution Features of SOC Content. The organic matter in the soil interacts with the engineering polymer to a certain extent, and the degradation of the artificial polymer is induced where the organic matter exists. Organic matter is one of the important reasons for the degradation of artificial macromolecules. The microplastics discharged into the environment will interact with the organic matter in the soil. The addition of humus in the soil will increase the steric hindrance between the microplastics themselves, making the particle size of the microplastics significantly smaller than that in the presence of humic acid. Therefore, when humic acid exists, the penetration ability of the microplastics is enhanced, and its retention in the medium is reduced. Understanding soc content in Beijing Plain is helpful for remediation of soil macromolecular pollution in the later period.

We calculated the statistical eigenvalues of SOC content in different years. The Shapiro-Wilk test (the W method) is used for normal distribution test.

The mean value of SOC content increased with time. According to variance analysis, there was a significant difference $(F=3.042, d f=727, p \leqslant 0.001)$ in SOC content during the six years. As the mean value of SOC content increased, the standard deviation and range also increased; i.e., the regional difference became increasingly significant with the human interference. The different interannual SOC contents were consistent with the linear relationship, and the fitting effect was significant $\left(d f=4, R^{2}=0.8733>R^{2}{ }_{0.05}=0.88114\right)$. According to the normal distribution test of the $\mathrm{W}$ method, the SOC contents from different years were consistent with the normal distribution $(\mathrm{p}>0.05)$. The cumulative variation ratio of farmland SOC content in the plain areas of Beijing was $12.89 \%$, with an average annual variation ratio of $2.58 \%$
(Table 1). In the literature conducted by Liang et al. [30] found that the average farmland SOC content of North China was $17.70 \mathrm{~g} \cdot \mathrm{kg}^{-1}$, which is higher than the maximum observed in this study. The above results indicate that there is still huge potential for increases in the farmland SOC content.

The EBK method was then applied to obtain the SOC temporal stability variation coefficient figure (Figure 3), which shows the time-based variation trend of SOC content. The subset of the empirical Bayesian method was 100; the number of overlap factors was 3 ; the number of simulations was 100; there were 4 search sections; the search radius was $29.55 \mathrm{~km}^{2}$; and the output grid was $100 \mathrm{~m} \times 100 \mathrm{~m}$ (the size of lattice was $1 \mathrm{ha}^{2}$ ).

According to Figure 3, the average of SOC temporal stability coefficients in the research zones was 20.25. The SOC temporal stability mainly stayed in $M S 3$, between stability and instability. The areas of $M S_{1}, M S_{2}$, and $M S_{3}$ were 42.29 $\mathrm{km}^{2}, 1027.14 \mathrm{~km}^{2}$, and $718.11 \mathrm{~km}^{2}$, respectively. The unstable areas were mainly located in Haitian District, Chaoyang District, Sheringham District, and Changing District in the urban life area. The temporal stability of SOC content was totally different from the SOC content in terms of spatial distribution, and regions with higher SOC content showed a lower temporal stability.

As far as functional area is concerned, SOC content was the least stable in the urban life area and the peripheral circle with a radius of $35 \mathrm{~km}$. For over $80 \%$ of the urban life area, temporal stability stayed in US. In contrast, the agricultural security area and the water source conservation exhibited opposite patterns, and these areas mainly stayed in MS.

3.2. Analysis of Influencing Factors for the Evolution of SOC Content. This study selects factors that reflect farmland management such as the input of organic fertilizer and the plantation system as well as the soil texture of the natural environment to analyze the driving mechanism behind the evolution of farmland SOC content.

3.3. Input of Organic Fertilizer. From 2010 to 2015, the average annual fertilizer input was $38.24 \mathrm{~kg} \cdot \mathrm{ha}^{-1}, 38.16 \mathrm{~kg} \cdot \mathrm{ha}^{-1}$, $38.81 \mathrm{~kg} \cdot \mathrm{ha}{ }^{-1}, 40.43 \mathrm{~kg} \cdot \mathrm{ha}^{-1}, 42.25 \mathrm{~kg} \cdot \mathrm{ha^{-1 }}, 44.20 \mathrm{~kg} \cdot \mathrm{ha^{-1 }}$, respectively. The correlation analysis between the input of organic fertilizer (scalar quantity, $\mathrm{kg} \cdot \mathrm{ha}^{-1}$ ) and the mean SOC content of corresponding years is carried out (Figure 4(a)). $R$ at the annual scale was employed to calculate the spatial correlation (Figure 4(b)). 


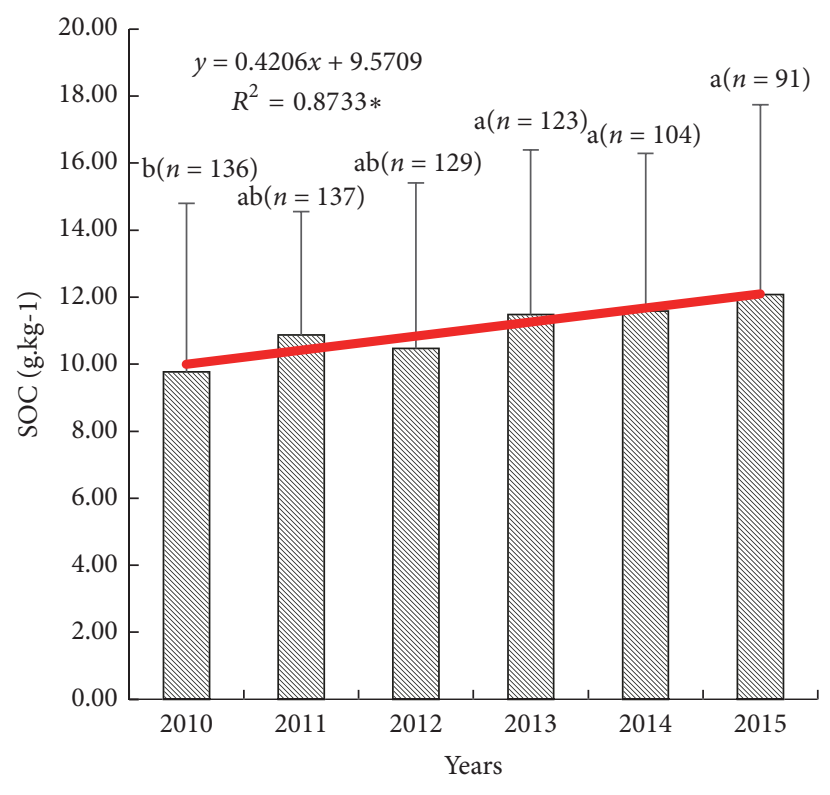

(a) Histogram of soil organic carbon

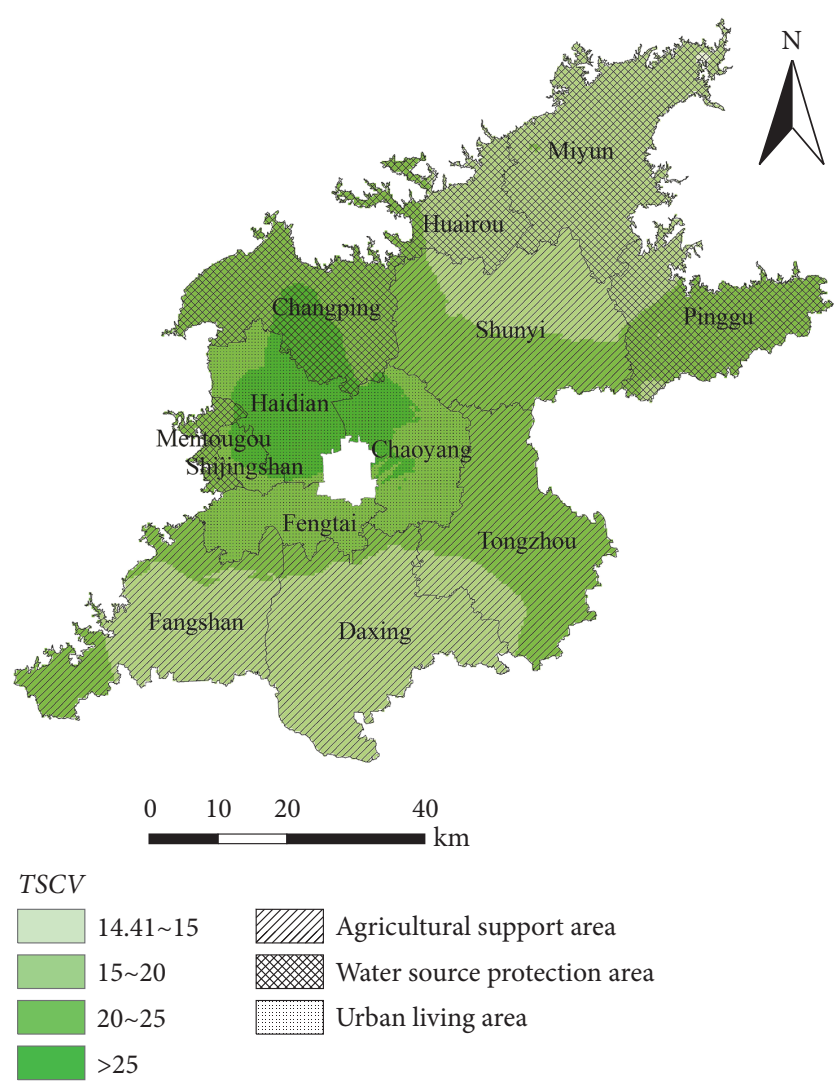

(b) Diagram of time stability coefficient

FIgURE 3: The interannual variation and spatial distribution of soil organic carbon and its trend. Values in each row with the same letter are not significant $(P>0.05)$. In the equation, $x$ and $y$, respectively, represent the year number $(1,2, \ldots, 6)$ and soil organic carbon content.

Figure 4(a) shows the quantitative fitting relationship between the mean SOC contents and the input of organic fertilizer (scalar quantity) during the 6 years. The average SOC content and the input of fertilizer were consistent with a linear equation, and the fit is significant $\left(d f=4, R^{2}=0.8433\right.$ $\left.<R_{0.05}^{2}=0.88114\right)$. An increase in the input of organic fertilizer led to an increase in SOC content, and an extra unit $\left(1 \mathrm{~kg} \cdot \mathrm{ha}^{-1}\right)$ of organic fertilizer led to an increase of 0.057 $\mathrm{g} \cdot \mathrm{kg}^{-1}$ in SOC content.

The average spatial correlation coefficient between SOC content and the input of organic fertilizer over several years was 0.49 (Figure 4(b)), and there was an overall positive spatial correlation between these two variables. The regions with a positive correlation had a total area of $6120.5 \mathrm{~km}^{2}$, accounting for $78.69 \%$ of the total study area. Specifically, $19.72 \%$ and $31.30 \%$ of the total area exhibited significance at the $p<0.05$ level and the $p<0.01$ level, respectively. The input of organic fertilizer can effectively increase SOC content. The above conclusions are consistent with the achievements of relevant studies (Dendoncker et al., 2011). According to the studies of Shen et al. [31], Chen et al. [32], Wu et al. [33], Dendoncker (2011), and Liang et al. [34], the average carbon fixation rate of the farmland soil in Belgium nearly doubled with the use of organic fertilizer. Shen et al. [31] also argued that the long-term use of organic fertilizer or a mixture of organic fertilizer and NPK mineral fertilizer helped increase total SOC content. Chen et al. [32] found that long-term use of organic fertilizer could promote the continual growth of soil organic content and that the extent of growth rose with the increase in the input of organic fertilizer. An experiment by $\mathrm{Wu}$ et al. [33] indicated that organic fertilizer, especially the mixture of chemical fertilizer and organic fertilizer, could enhance SOC content.

3.4. Soil Texture. To quantify significant differences in SOC content among different years, 6 groups (6 years) of SOC content were used for the variance analysis. According to Levine's homogeneity of variance test, the mean values of SOC content were all consistent with the homogeneity of variance. Therefore, the least significant difference method was adopted.

Throughout the 6 years, the content of organic carbon in different soil texture is medium loam, light loam, sandy loam, and sandy soil from high to low (Table 2). There was a significant difference among different types of soil texture in the same year. There was an extremely significant difference in SOC content between sandy soil and medium loam as well as other types of soil texture $(\mathrm{p} \leqslant 0.01)$. This indicates that soil with greater adhesion has higher organic carbon (organic) 


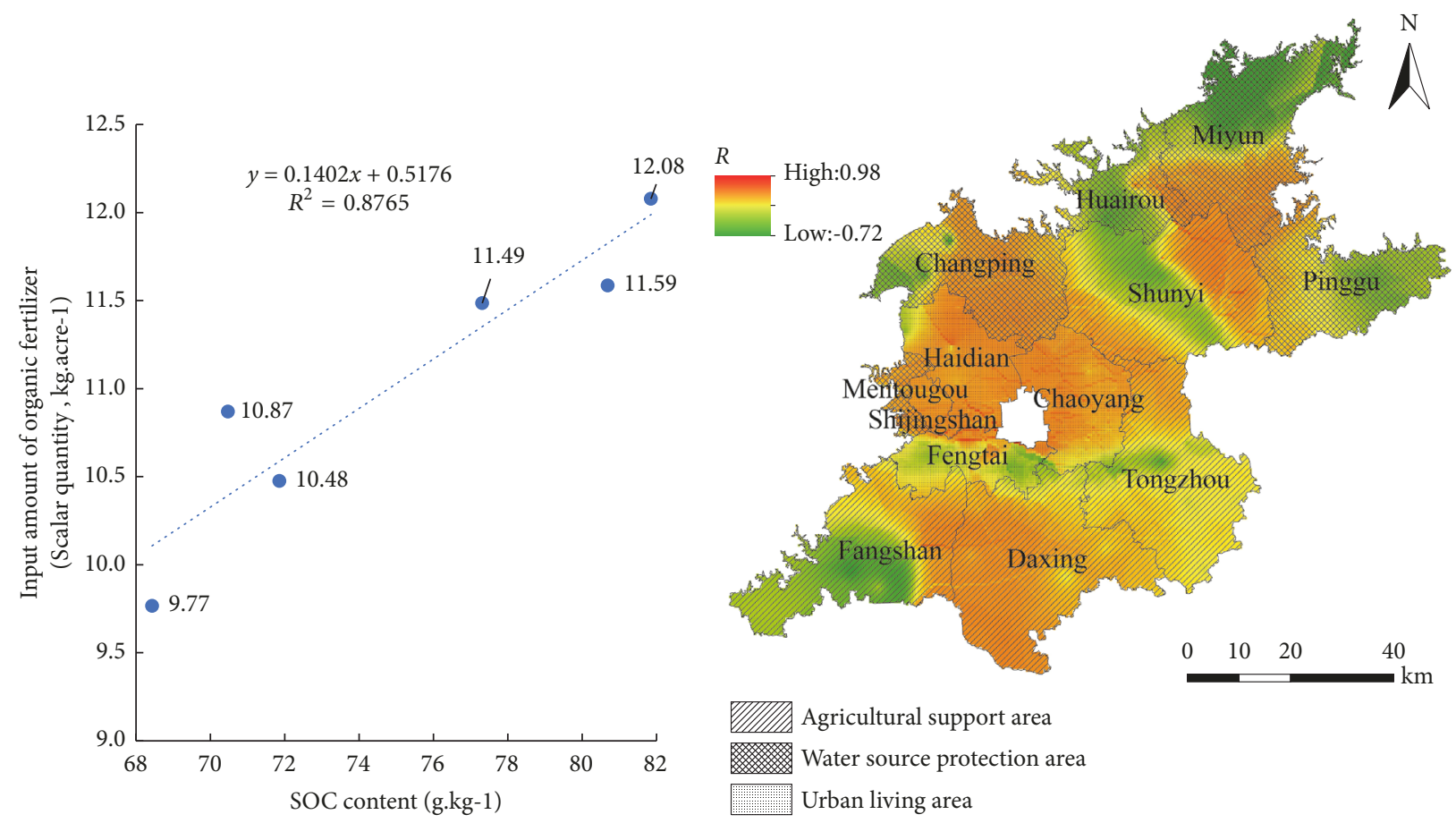

(a) Amount relationship between soil organic carbon content and input amount of organic fertilizer

(b) Spatial correlation between soil organic carbon content and input amount of organic fertilizer

FIGURE 4: The relationship between soil organic carbon and the input of organic fertilizer. Values in each row with the same letter are not significant $(P>0.05)$. In the equation, $x$ and $y$, respectively, indicated soil organic carbon content and input amount of organic fertilizer.

TABLE 2: The soil organic carbon content of variant soil textures in different years.

\begin{tabular}{|c|c|c|c|c|c|c|c|}
\hline \multirow{2}{*}{ Soil texture types } & \multicolumn{6}{|c|}{$\mathrm{SOC} / \mathrm{g} \cdot \mathrm{kg}^{-1}$} & \multirow{2}{*}{ Amplitude/\% } \\
\hline & 2010 & 2011 & 2012 & 2013 & 2014 & 2015 & \\
\hline Middle loam & $10.44 \pm 3.82 \mathrm{~b}$ & $12.77 \pm 4.81 \mathrm{a}$ & $12.21 \pm 7.45 \mathrm{a}$ & $13.84 \pm 4.92 \mathrm{a}$ & $14.34 \pm 7.53 \mathrm{a}$ & $14.75 \pm 7.28 \mathrm{a}$ & 8.26 \\
\hline Light loam & $11.15 \pm 3.92 \mathrm{a}$ & $11.40 \pm 3.16 \mathrm{a}$ & $10.89 \pm 3.68 b$ & $11.55 \pm 4.02 \mathrm{a}$ & $11.67 \pm 3.28 \mathrm{a}$ & $12.48 \pm 5.65 b$ & 2.39 \\
\hline Sandy loam & $10.56 \pm 5.20 \mathrm{~b}$ & $9.44 \pm 3.53 \mathrm{~b}$ & $9.50 \pm 5.78 c$ & $10.76 \pm 6.31 b$ & $10.07 \pm 4.54 \mathrm{~b}$ & $10.36 \pm 3.54 c$ & -0.39 \\
\hline Sand & $8.44 \pm 2.78 b$ & $8.32 \pm 2.65 b$ & $7.77 \pm 2.99 \mathrm{c}$ & $8.07 \pm 3.46 \mathrm{~b}$ & $10.43 \pm 4.66 b$ & $9.58 \pm 5.39 c$ & 2.70 \\
\hline
\end{tabular}

Note: values with same letter in the same year indicates that the SOC of different soil texture is not significantly different with time ( $\mathrm{p}>0.05)$.

content. This result is consistent with the conclusions of previous studies [35-37].

The SOC content of medium and light loams generally increased with time, and there was a significant interannual difference in the SOC content $(F=0.08, p<0.05)$. The average extent of increase in the SOC content of medium loam was $8.26 \%$, and the SOC exceeded $15 \mathrm{~g} \cdot \mathrm{kg}^{-1}$ by 2015 . The SOC content of medium loam increased by $2.39 \%$ on average, and the SOC approached $14 \mathrm{~g} \cdot \mathrm{kg}^{-1}$ by 2015 . The SOC content of was relatively low at approximately $10 \mathrm{~g} \cdot \mathrm{kg}^{-1}$. There was no interannual difference in the SOC for sandy loam and sandy soil, and the SOC content of sandy loam declined overall. The standard deviation of SOC for all soil textures exhibited an increasing trend. This indicates that the regional SOC content depends on human and natural effects.

3.5. Plantation System. Pesticides play an important role in agricultural production, but due to the toxicity of pesticides, such as ecological damage, water, soil, and air pollution. Pesticide residues refer to various substances such as pesticide protoplasts, toxic metabolites, and degradants that remain in agricultural products after the use of pesticides. Pesticides in farmland belong to macromolecule pollutants, and organic matter, especially humus, can degrade pesticides.

The plantation systems deployed in our study area includes vegetable fields (protected and open ones), grain fields, and orchards, and these substantially differ in terms of agricultural management.

Figure 5 shows the average soil organic carbon content in different crop cropping systems and its change over time. In general, the mean SOC content of greenhouse vegetable field, open vegetable field, grain field, and orchard field were 13.03, $12.26,11.02$, and $10.06 \mathrm{~g} \cdot \mathrm{kg}^{-1}$, respectively during 2010 to 2015 . And we can see that soil organic carbon content in different crop systems in the same year had significant differences except 2010 and 2013. On the other hand, the SOC content 


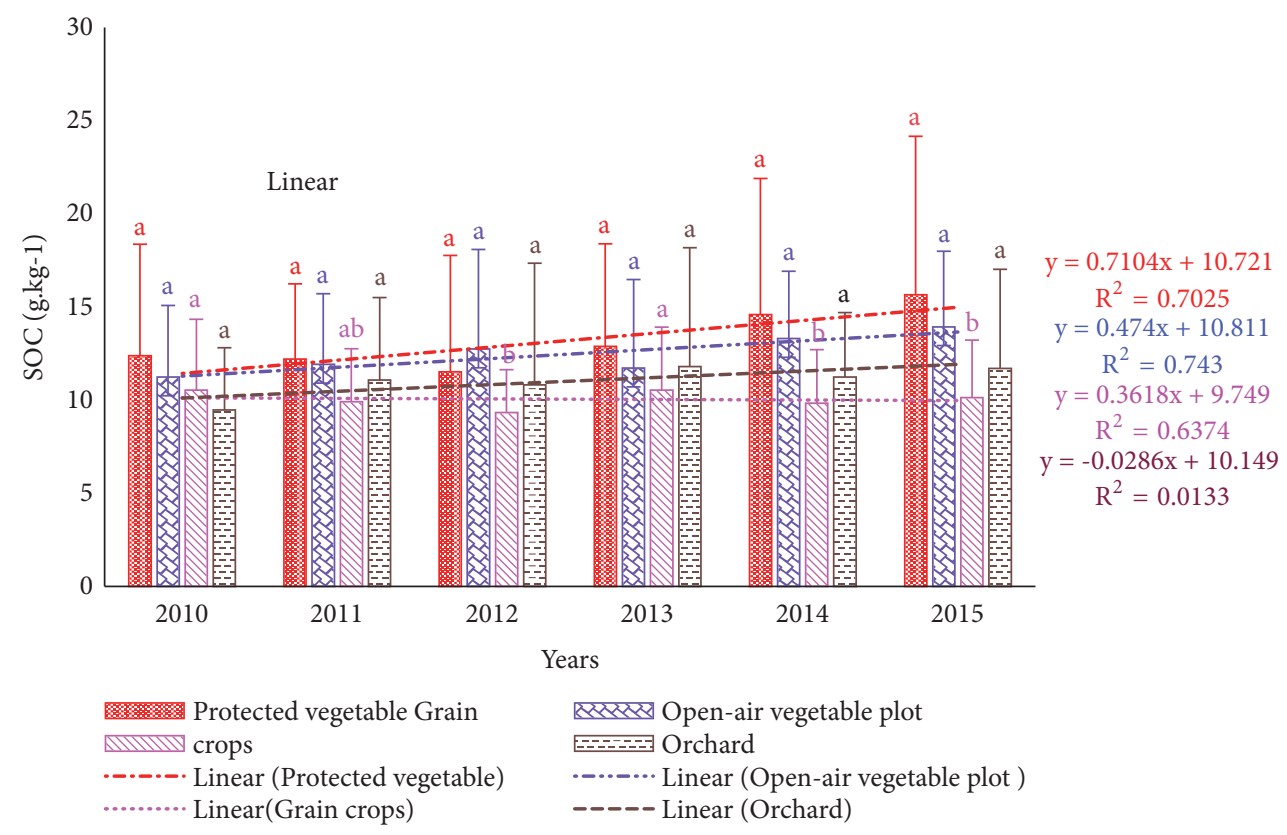

FIGURE 5: The SOC content in different planting system and different year. Values with same letter in the same planting system indicate that the SOC is not significant different with time $(\mathrm{p}>0.05)$. In the equation, $\mathrm{x}$ and $\mathrm{y}$, respectively, indicated the year number $(1,2, \ldots, 6)$ and soil organic carbon content.

of the same crop planting system varies with time. Firstly, the mean values of SOC content for the greenhouse vegetable fields from 2010 to 2015 were $(12.39 \pm 5.98)$ a, $(12.21 \pm 4.03)$ a, $(11.51 \pm 6.25)$ a, $(12.88 \pm 5.51)$ a, $(14.59 \pm 7.31)$ a, and $(15.66 \pm 8.50)$ a g.kg-1, respectively. With the year of 2013 as the inflection point, the average SOC content of protected vegetable fields first declined and then increased throughout the 6 years. The trend was consistent with the linear equation, and the correlation coefficient $\left(R^{2}\right)$ was 0.7025 . Secondly, the mean value of SOC content of open vegetable fields from 2010 to 2015 were $(11.23 \pm 3.85)$ a, $(11.91 \pm 3.80)$ a, $(12.73 \pm 5.35)$ a, $(11.71 \pm 4.76)$

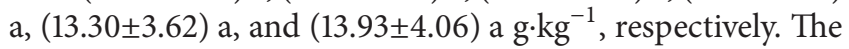
trend was consistent with the linear equation as a whole, and the R2 was 0.7430 . Thirdly, the mean values of SOC content of orchards from 2010 to 2015 were $(9.46 \pm 3.35)$ a, $(11.07 \pm 4.43)$ a, $(10.82 \pm 6.53)$ a, $(11.81 \pm 6.37)$ a, $(11.24 \pm 3.46)$ a, and $(11.69 \pm 5.33)$ a $\mathrm{g} \cdot \mathrm{kg}^{-1}$, respectively. The SOC content of orchards showed an overall increasing trend. This trend was consistent with the linear fitting equation, with a correlation coefficient of 0.6734. Compared with other plantation systems, the SOC variation of orchards was relatively stable, and the SOC content remains approximately $11 \mathrm{~g} \cdot \mathrm{kg}-1$. Lastly, the mean values of SOC content in the grain fields from 2010 to 2015 were $(10.54 \pm 3.80) \mathrm{a},(9.92 \pm 2.84) \mathrm{a},(9.32 \pm 2.31) \mathrm{a},(10.54 \pm 3.38)$ a, $(9.84 \pm 2.87)$ a, and $(10.14 \pm 3.08)$ a g.kg-1, respectively. The variation of average SOC content of grain fields during the 6 years was relatively irregular and showed and overall trend of decline. The trend fitted the linear equation, showing a negative correlation.

The mean value of several years and interannual variation of the SOC content of different plantation systems showed the following pattern: protected vegetable fields $>$ open vegetable fields $>$ orchards $>$ grain fields. The variation of SOC content can be roughly divided into two types. The first type showed a tendency of increase, including protected vegetable fields, open vegetable fields, and orchards, and the second type showed an overall tendency of decline, i.e., grain fields. Protected vegetable fields feature high input, high output, and multiple-cropping systems, and this is significantly different from the ecological environment of uncultivated soil. Grain fields are characterized with a low level of inputs, and most of the fertilizers were inorganic. Orchards feature high but singular input, and the management mode remained largely unchanged. In recent years, Beijing has published such documents as the Beijing Municipal Plan for the Development of Modern Agriculture during the 12th "Five-Year Plan" Period (2011-2015) and Opinions on Promoting the Development of Protected Agriculture to increase the input for protected agriculture and orchards. By 2020, the area of grain fields, vegetable fields, and orchards in Beijing will reach 53,333ha, 46,667ha, and 66,667ha, respectively, showing a "ThreePillar" pattern. As the area of grain fields declines and the area of vegetable fields and orchards increases, corresponding inputs should be enhanced.

\section{Conclusions}

In this study, long-term monitoring at fixed locations in conjunction with the temporal stability coefficient, the regression slope, the EBK, spatial correlation analysis, and variance analysis were used to reveal the evolution character of the SOC content and relevant driving mechanisms in the plain areas of Beijing. The main conclusions are listed as follows: 
(1) There was significant variance in the mean SOC content during the 6-year period and in general, the SOC content increases with time. (2)As a whole, the temporal stability of SOC was moderately stable. However, it was completely different from the spatial distribution of SOC. Specifically, the temporal stability of SOC is low in regions with high SOC content. (3) The application of organic fertilizer can significantly increase the soil organic carbon content. This study showed that the organic carbon content increased by $0.057 \mathrm{~g} \cdot \mathrm{kg}^{-1}$ for every 1 unit of organic fertilizer. (4) The grain field plantation system exhibited declining SOC content, but protected vegetable fields, open vegetable fields, and orchards all showed an increase in SOC content. (5) Regional SOC content depends on human and natural effects with human factor playing a dominant role, although soil with a higher clay content exhibits higher SOC content. (6) As a kind of polymer pollutant, engineering polymer has influence on soil properties. Clarifying the influence and mechanism of organic matter on the adsorption and migration behavior of engineering polymers in water-soil multimedia environment, and the distribution of SOC in Beijing plain area are helpful for remediation of farmland pollution in the later period.

\section{Data Availability}

The authors declare that the data supporting the findings of this study are available within the article.

\section{Conflicts of Interest}

The authors declare that they have no conflicts of interest.

\section{Acknowledgments}

This work was supported by the National Key Research and Development Program of China (2016YFD0300801).

\section{References}

[1] P. Biswas and C. Y. Wu, "2005 critical review: nanoparticles and the environment," Joumal of the Air and Waste Management Association, vol. 55, pp. 708-746, 2005.

[2] B. Nowack and T. D. Bucheli, "Occurrence, behavior and effects of nanoparticles in the environment," Environmental Pollution, vol. 150, no. 1, pp. 5-22, 2007.

[3] P. G. Tratnyek and R. L. Johnson, "Nanotechnologies for environmental cleanup," Nano Today, vol. 1, no. 2, pp. 44-48, 2006.

[4] B. K. Theng and G. Yuan, "Nanoparticles in the soil environment," Elements, vol. 4, no. 6, pp. 395-399, 2008.

[5] M. F. Hochella, S. K. Lower, P. A. Maurice et al., "Nanominerals, mineral nanoparticles, and earth systems," Science, vol. 319, no. 5870, pp. 1631-1635, 2008.

[6] P. K. Pranzas, R. Willumeit, R. Gehrke, J. Thieme, and A. Knochel, "Characterisation of structure and aggregation processes of aquatic humic substances using small-angle scattering and X-ray microscopy," Analytical and Bioanalytical Chemistry, vol. 376, no. 5, pp. 618-625, 2003.
[7] J. N. Ryan and M. Elimelech, "Colloid mobilization and transport in groundwater," Colloids and Surfaces A: Physicochemical and Engineering Aspects, vol. 107, pp. 1-56, 1996.

[8] A. J. Pelley and N. Tufenkji, "Effect of particle size and natural organic matter on the migration of nano- and microscale latex particles in saturated porous media," Journal of Colloid and Interface Science, vol. 321, no. 1, pp. 74-83, 2008.

[9] T. Phenrat, J. E. Song, C. M. Cisneros, D. P. Schoenfelder, R. D. Tilton, and G. V. Lowry, "Estimating attachment of nano- and submicrometer-particles coated with organic macromolecules in porous media: Development of an empirical model," Environmental Science \& Technology, vol. 44, no. 12, pp. 4531-4538, 2010.

[10] B. Chefetz and B. Xing, "Relative role of aliphatic and aromatic moieties as sorption domains for organic compounds: a review," Environmental Science \& Technology, vol. 43, no. 6, pp. 1680$1688,2009$.

[11] D. Grasso, K. Subramaniam, J. Pignatello, Y. Yang, and D. Ratté, "Micellar desorption of polynuclear aromatic hydrocarbons from contaminated soil," Colloids and Surfaces A: Physicochemical and Engineering Aspects, vol. 194, no. 1-3, pp. 65-74, 2001.

[12] A. Ussawarujikulchai, S. Laha, and B. Tansel, "Synergistic effects of organic contaminants and soil organic matter on the soilwater partitioning and effectiveness of a nonionic surfactant (triton x-100)," Bioremediation Journal, vol. 12, no. 2, pp. 88-97, 2008.

[13] C. Li, Y. Zhuang, S. Frolking et al., "Modeling soil organic carbon change in croplands of China," Ecological Applications, vol. 13, no. 2, pp. 327-336, 2003.

[14] P. Falloon, P. Smith, R. I. Bradley et al., "RothCUK - A dynamic modelling system for estimating changes in soil C from mineral soils at 1-km resolution in the UK," Soil Use and Management, vol. 22, no. 3, pp. 274-288, 2006.

[15] S. M. Ogle, F. J. Breidt, M. Easter, S. Williams, K. Killian, and K. Paustian, "Scale and uncertainty in modeled soil organic carbon stock changes for US croplands using a process-based model," Global Change Biology, vol. 16, no. 2, pp. 810-822, 2010.

[16] G. Wang, Y. Huang, E. Wang, Y. Yu, W. Zhang, and B. Bond-Lamberty, "Modeling soil organic carbon change across australian wheat growing areas, 1960-2010," Plos One, vol. 8, no. 5, Article ID e63324, 2013.

[17] C. Li, S. Frolking, and R. Harriss, "Modeling carbon biogeochemistry in agricultural soils," Global Biogeochemical Cycles, vol. 8, no. 3, pp. 237-254, 1994.

[18] S. D. Jenkinson, P. S. Andrew, M. J. Lynch, J. M. Goss, and P. B. Tinker, "The turnover of organic carbon and nitrogen in soil," Philosophical Transactions of the Royal Society B Biological Sciences, vol. 329, no. 329, pp. 361-367, 1990.

[19] W. J. Parton, J. M. Scurlock, D. S. Ojima et al., "Observations and modeling of biomass and soil organic matter dynamics for the grassland biome worldwide," Global Biogeochemical Cycles, vol. 7, no. 4, pp. 785-809, 1993.

[20] B. A. Keating, P. S. Carberry, G. L. Hammer et al., "An overview of APSIM, a model designed for farming systems simulation," European Journal of Agronomy, vol. 18, no. 3-4, pp. 267-288, 2003.

[21] J. Guo, Z. Song, X. Peng et al., "Evaluation in soil carbon and nitrogen characteristics under long-term cropping regimes in black soil region of Northeast China," Nongye Gongcheng Xuebao, vol. 31, no. 6, pp. 178-185, 2015. 
[22] E. A. Davidson and I. L. Ackerman, "Changes in soil carbon inventories following cultivation of previously untilled soils," Biogeochemistry, vol. 20, no. 3, pp. 161-193, 1993.

[23] T. O. West and G. Post, "Soil organic carbon sequestration rates by tillage and crop rotation: a global data analysis," Soil Science Society of America Journal, vol. 66, no. 6, pp. 1930-1946, 2002.

[24] G. Wang, Z. Luo, E. Wang, and Y. Huang, "Contrasting effects of agricultural management on soil organic carbon balance in different agricultural regions of China," Pedosphere, vol. 23, no. 6, pp. 717-728, 2013.

[25] G. Erdem, Ç. Sağır, M. Canoglu C, and B. Kurtulus, "Comparison of empirical bayesian kriging and geo- anfis methods for interpolating hydraulic head in a karst alluvium," in Proceedings of the Iemss 2016 - International Congress on Environmental Modelling and Software, 2016.

[26] K. M. Mwenda, "Quantifying uncertainty of spatial interpolation of fine particulate matter in small regions using empirical bayesian kriging," in Proceedings of the International Chemical and Environmental Engineering Conference Iceec, 2014.

[27] A. Gribov and K. Krivoruchko, "New flexible non-parametric data transformation for trans-gaussian kriging," in Geostatistics Oslo 2012, vol. 17 of Quantitative Geology and Geostatistics, pp. 51-65, Springer Netherlands, Dordrecht, 2012.

[28] K. Krivoruchko, Spatial Statistical Data Analysis for GIS Users, 2011.

[29] S. Blackmore, "The interpretation of trends from multiple yield maps," Computers and Electronics in Agriculture, vol. 26, no. 1, pp. 37-51, 2000.

[30] E. Liang, Y. Cai, C. Dai, D. Zhang C, Z. Feng H, and S. Liu, "Change of soil organic carbon in cropland of China. Analysis of driving factors," Chinese Soil and Fertilizer, no. 6, pp. 80-86, 2010.

[31] H. Shen, H. Z. Cao, and Z. H. Xu, "Effects of Fertilization on different forms of soil carbon and carbon pool management index," Journal of the Chinese society of soil science, vol. 37, no. 2, pp. 166-173, 2000.

[32] Y. Chen, C. Y. Wu, and J. G. Shui, "Emission and fixation of $\mathrm{CO}_{2}$ from soil system as influenced by long-term application of organic manure in paddy soils," Agricultural Sciences in China, vol. 5, no. 6, pp. 456-461, 2006.

[33] T. Wu, J. J. Schoenau, F. Li et al., "Influence of cultivation and fertilization on total organic carbon and carbon fractions in soils from the Loess Plateau of China," Soil \& Tillage Research, vol. 77, no. 1, pp. 59-68, 2004.

[34] B. Liang, X. Yang, X. He, D. V. Murphy, and J. Zhou, "Long-term combined application of manure and NPK fertilizers influenced nitrogen retention and stabilization of organic C in Loess soil," Plant and Soil, vol. 353, no. 1-2, pp. 249-260, 2012.

[35] K. Hu, H. Li, B. Li, and Y. Huang, "Spatial and temporal patterns of soil organic matter in the urban-rural transition zone of Beijing," Geoderma, vol. 141, no. 3-4, pp. 302-310, 2007.

[36] W. S. Zhang, R. H. Ning, L. D. Xu, Y. Huichun, Y. H. Zhang, and Y. F. Huang, "Spatio-temporal evolution and driving factors of vegetation cover in open pit coal mine of grassland area," Transactions of the Chinese Society of Agricultural Engineering, vol. 32, no. 17, pp. 233-241, 2016.

[37] D. McGrath and C. Zhang, "Spatial distribution of soil organic carbon concentrations in grassland of Ireland," Applied Geochemistry, vol. 18, no. 10, pp. 1629-1639, 2003. 


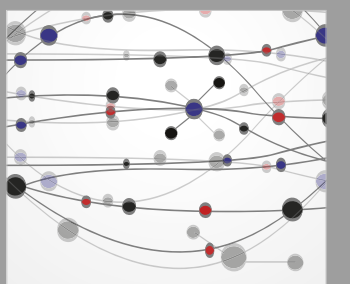

The Scientific World Journal
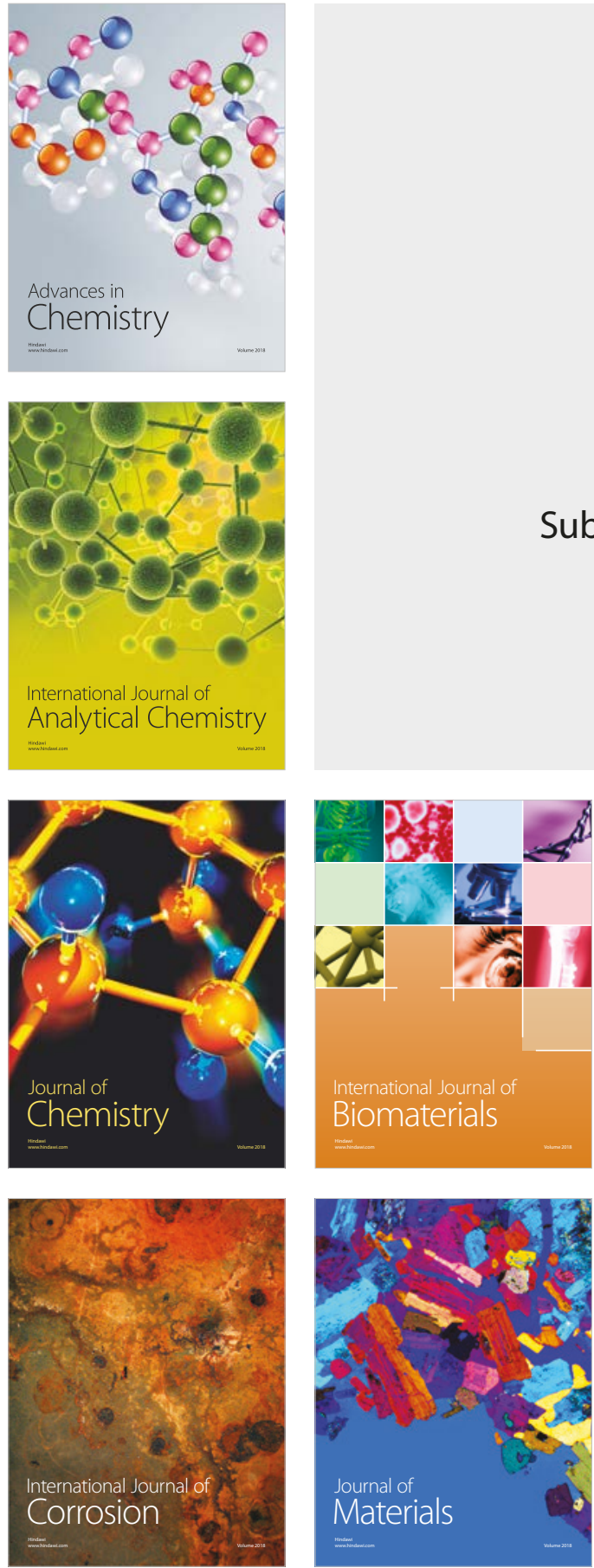

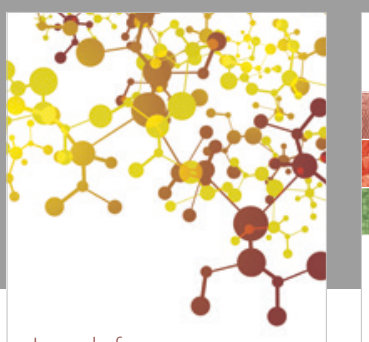

Journal of

Applied Chemistry
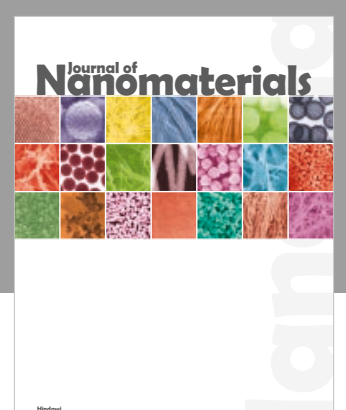

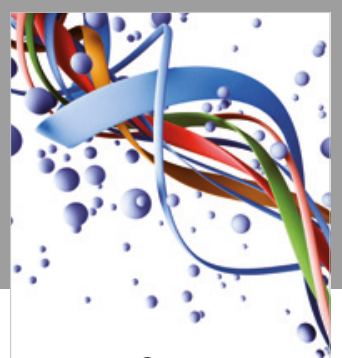

Scientifica

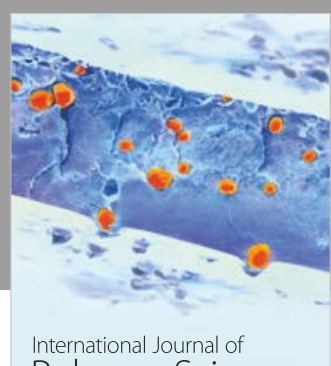

Polymer Science

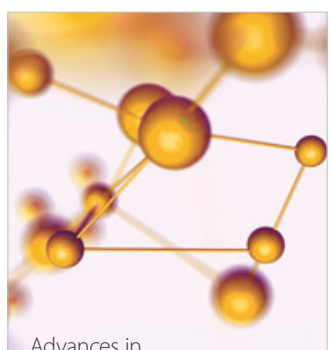

Physical Chemistry
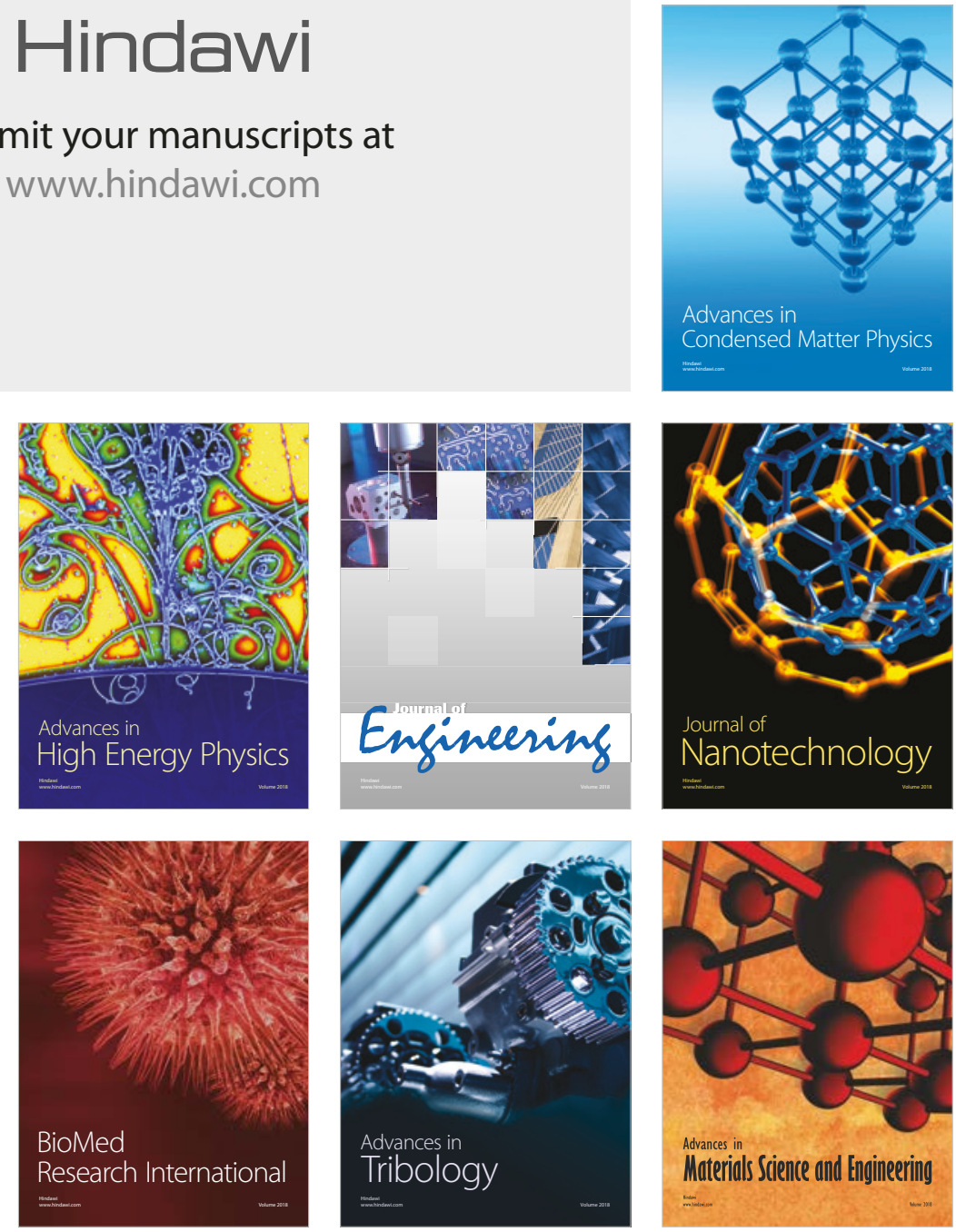